Gregory Martin ORCID iD: 0000-0002-7099-7913

\title{
The assimilation of glycerol into lipid acyl chains and associated carbon backbones of Nannochloropsis salina varies under nitrogen replete and deplete conditions
}

Nature Poddar ${ }^{1}$, Sheik Nadeem Elahee Doomun ${ }^{2}$, Damien L. Callahan ${ }^{2}$, Greg M Kowalski ${ }^{3}$,

\section{Gregory J. O. Martin ${ }^{1 *}$}

${ }^{1}$ Algal Processing Group, Department of Chemical Engineering, The University of Melbourne, Parkville, Victoria 3010, Australia.

${ }^{2}$ School of Life and Environmental Science, Deakin University, Burwood, Victoria 3125, Australia.

${ }^{3}$ Institute for Physical Activity and Nutrition, School of Exercise Sciences, Deakin, Geelong, Australia.

\section{Short running title:}

Glycerol assimilation into N. salina acyl lipids

\section{*Corresponding author:}

Gregory J.O. Martin

Address: Department of Chemical Engineering, The University of Melbourne, Parkville 3010 Victoria, Australia

Telephone: +613 83446613

This is the author manuscript accepted for publication and undergone full peer review but has not been through the copyediting, typesetting, pagination and proofreading process, which may lead to differences between this version and the Version of Record. Please cite this article as doi: 10.1002/bit.27498. 
Email: gjmartin@unimelb.edu.au

\begin{abstract}
Mixotrophic cultivation can increase microalgae productivity, yet the associated lipid metabolism remains mostly unknown. Stable isotope labelling was used to track assimilation of glycerol into the TAG and membrane lipids of Nannochloropsis salina. In N-replete media, glycerol uptake and ${ }^{13} \mathrm{C}$ incorporation into acyl chains were respectively 6-fold and 12-fold higher than in $\mathrm{N}$-deplete conditions. In N-replete cultures, $42 \%$ of the carbon in the consumed glycerol was assimilated into lipid acyl chains, mostly in membrane lipids rather than TAG. In N-deplete cultures, only $11 \%$ of the limited amount of consumed glycerol was fixed into lipid acyl chains. Labelled lipid-associated glycerol backbones were predominantly ${ }^{13} \mathrm{C}_{3}$ labelled, suggesting that intact glycerol molecules were directly esterified with fatty acids/polar head groups. However, the presence of singly and doubly labelled lipid-bound glycerol species suggested that some glycerol also went through the central carbon metabolism prior to forming glycerol-3-phosphate destined for lipid esterification. ${ }^{13} \mathrm{C}$ incorporation was higher in the saturated and monounsaturated than the polyunsaturated acyl chains of TAG, indicating the flux of carbon from glycerol went firstly to de novo fatty acid synthesis before acyl editing reactions. The results demonstrate that nitrogen availability influences both glycerol consumption and utilisation for lipid synthesis in Nannochloropsis, providing novel insights for developing mixotrophic cultivation strategies.
\end{abstract}

Keywords: Mixotrophy; microalgae; lipid synthesis; ${ }^{13} \mathrm{C}_{3}$ glycerol; nitrogen deprivation; GC-MS

This article is protected by copyright. All rights reserved. 


\section{Introduction}

Nannochloropsis sp. is an industrially important microalga that can produce high amounts of triacylglyceride (TAG) and eicosapentaenoic acid (EPA; a long chain polyunsaturated fatty acid) (Wang et al., 2014). TAG accumulation in this alga can be induced following nutrient limitation, for instance nitrogen deprivation (Martin et al., 2014). In Nannochloropsis sp., the EPA fatty acids are primarily associated with the membrane lipids, particularly the glycolipids and phospholipids (Martin et al., 2014; Simionato, Basso, Giacometti, \& Morosinotto, 2013). The use of mixotrophic growth on glycerol can result in higher algal biomass and lipid productivities compared to photoautotrophic regime (Deschenes, Boudreau, \& Tremblay, 2015; Paranjape, Leite, \& Hallenbeck, 2016a, 2016b; N. Poddar, Sen, \& Martin, 2018). However, the combined effect of glycerol addition and nitrogen deprivation on TAG accumulation has not been explored in detail (Pagnanelli, Altimari, Trabucco, \& Toro, 2014). It is not clear how the carbon from glycerol is partitioned between TAG and membrane lipids, between acyl chains and glycerol backbones, or if nitrogen availability influences its fate.

The use of isotope labelled carbon sources has provided a means of investigating carbon metabolism in algae. Previous studies have analysed the carbon flux using radioactive or stable isotope labelling techniques and by following the kinetics of a labelled substrate at various stages of metabolism (Allen, DiRusso, \& Black, 2017; Bates \& Browse, 2012; Bates, Durrett, Ohlrogge, \& Pollard, 2009; Villanova et al., 2017). Stable isotope labelling enables detailed investigations into the metabolism of carbon using mass spectrometry based analytical techniques (Elahee Doomun, Loke, O'Callaghan, \& Callahan, 2016). During growth, microalgae direct carbon towards 
intracellular components such as proteins, cell walls, and membrane lipids such as phospholipids and glycolipids. Nitrogen deplete conditions divert metabolism from growth, towards the accumulation of intracellular carbon-rich energy reserves such as TAG. Stable isotope labelled enrichment studies have been used previously to determine the origin of the fatty acyl chains of TAG as a function of environmental conditions such as nitrogen availability (Janssen, Lamers, de Vos, Wijffels, \& Barbosa, 2019). The de novo synthesis of fatty acids can be detected by isotope label enrichment on saturated (C16:0/C18:0) and monounsaturated (C16:1/C18:1) acyls chains (Ecker \& Liebisch, 2014), which are the precursors of longer, and more unsaturated fatty acids that are produced via acyl editing reactions (involving turnover of membrane lipids). As such, label enrichment on polyunsaturated fatty acid chains such as C20:5 can be used to detect membrane lipid turnover (Ecker \& Liebisch, 2014; Janssen et al., 2019; Zhang, Misra, Nargund, Coleman, \& Sriram, 2018). A study of the growth of Coccomyxa subellipsoidea on ${ }^{13} \mathrm{C}_{3}$-glucose, ${ }^{13} \mathrm{CO}_{2}$, or $\mathrm{D}_{2} \mathrm{O}$ under nitrogen limited conditions revealed that $80 \%$ of TAG was produced by de novo synthesis via the Kennedy pathway, while acyl chain transfer from membrane turnover only contributed approximately $20 \%$ of TAG (Allen et al., 2017). Meanwhile, ${ }^{13} \mathrm{C}$-sodium bicarbonate was used to demonstrate the translocation of $23 \%$ of EPA from polar lipids to TAG during nitrogen starvation in photoautotrophically grown N. gaditana (Janssen et al., 2019).

However, the assimilation pathways of glycerol into the microalgal lipidome are not yet fully understood. Previously, it has been shown that supplying exogenous glycerol can augment TAG production in diatoms (Villanova et al., 2017), yeast (Maruyama et al., 2018), and plant seeds (Pollard, Delamarter, Martin, \& Shachar-Hill, 2015). TAG is composed of three fatty acyl chains linked to a glycerol backbone (glycerol-3- 
phosphate, G3P) via ester linkages. Metabolically, de novo TAG can be directly produced from G3P via the Kennedy pathway. The sequence of acylation steps of G3P results in the subsequent synthesis of lysophosphatidic acid, phosphatidic acid, de novo diacylglycerol and TAG, completing the Kennedy pathway (Allen et al., 2017; Bates \& Browse, 2012; Xue, Chen, \& Jiang, 2017). Carbon flux analysis has revealed that the model diatom Phaeodactylum tricornutum can convert glycerol into G3P as the precursor to the glycerol backbone of TAG via the Kennedy pathway (Villanova et al., 2017). Alternatively, G3P can be funnelled into the gluconeogenesis pathway for carbohydrate production (Villanova et al., 2017). Subsequently, G3P can also be broken into acetyl-CoA that enters the tricarboxylic acid cycle (TCA) for amino acid synthesis (Huang, Liu, Yang, \& Wang, 2015). Of particular relevance to this study, acetyl-CoA is also a precursor of fatty acid synthesis.

Despite the advancement in lipidomic research using stable isotope labelled substrates, investigations into the metabolism of marine microalgae grown mixotrophically using glycerol remains limited. In particular, the impact of nitrogen availability on glycerol metabolism has not been investigated in relation to concurrent synthesis of G3P (incorporated as a TAG backbone) and TAG, and on acyl chain profile of glycerolipids in Nannochloropsis sp. Better understanding of the importance of glycerol for synthesis of TAG via mixotrophy requires tracking and quantifying the amount of glycerol carbon that partitions into the different lipid classes. This study aims to extend previous findings examining TAG and membrane lipid synthesis using isotopic labelling and mass-spectrophotometry techniques. Quantitative tracking of isotope labelled lipids recovered from $N$. salina grown mixotrophically under nitrate replete and deplete conditions enables analysis of acyl chain profiles within different lipid classes, particularly TAG. In the current study, $N$. 
salina was grown in the presence of uniformly labelled glycerol $\left({ }^{13} \mathrm{C}_{3}\right.$ glycerol $)$ in nitrate replete and deplete media for 7 days. GC-MS was used to quantify ${ }^{13} \mathrm{C}$ incorporation into the acyl chains and the glycerol backbone of the different lipid classes. This study, which is the first of its kind, provides new insight into the effect that mixotrophic growth on glycerol has on TAG and membrane lipid synthesis in $N$. salina, particularly under nitrogen deprivation.

\section{Materials and methods}

\subsection{Materials}

All chemicals used in this study were of HPLC grade. De-ionised water (18.3 M $\Omega$ ) was produced using a Milli- $\mathrm{Q}^{\mathrm{TM}}$ system. Plastic contamination was reduced by using $15 \mathrm{~mL}$ glass Kimble tubes with Teflon lids $\left(\mathrm{KIMAX}^{\circledR}\right.$, Thermo-Fisher Scientific, Australia), glass Pasteur pipettes, and Transferpettor positive displacement pipettes with glass tips (BRAND ${ }^{\circledR}$, Sigma-Aldrich) for all extraction steps. Kimble tubes were washed with methanol and were heated to $400{ }^{\circ} \mathrm{C}$ for $5 \mathrm{~h}$.

\subsection{Microalgae growth conditions}

Seed cultures of Nannochloropsis salina (CCMP 1776) were grown in $225 \mathrm{~cm}^{2}$ Corning Tissue Flasks ${ }^{\circledR}$ containing $400 \mathrm{~mL}$ of modified f/2 media (N. Poddar et al., 2018). Flasks were illuminated under a 14:10 hour light:dark cycle with a light intensity of $\sim 60 \mu \mathrm{mol}$ photons $\mathrm{m}^{-2} \mathrm{~s}^{-1}$ and mixed at $100 \mathrm{rpm}$ using a shaker (Unimax 1010, Heidolph, Australia).

\section{$2.3{ }^{13}$ C labelling growth conditions}

N. salina was labelled using ${ }^{13} \mathrm{C}_{3}$-glycerol (99\%, molecular weight of 95.07, Sigma Aldrich) in cultures maintained for 7 days. Seed cultures were inoculated at an initial 
cell concentration of $\sim 10^{7}$ cells $/ \mathrm{mL}$ in $30 \mathrm{~mL}$ nitrate replete $(1.17 \mathrm{mM})$ or deplete $(0$ mM) media supplied with 0.5 g/L (N. Poddar et al., 2018; Nature Poddar, Sen, \& Martin, 2019) of ${ }^{13} \mathrm{C}_{3}$-glycerol (6.15 mg of $\left.{ }^{13} \mathrm{C}\right)$. Cultures supplied with unlabelled glycerol were also incubated in parallel (Supplementary S.1). Ampicillin $(150 \mu \mathrm{g} / \mathrm{mL}$, Sigma Aldrich) was used to inhibit bacterial contamination. Biomass was harvested on the $7^{\text {th }}$ day, and the pellet freeze-dried for $48 \mathrm{~h}$. Cell-free supernatant was used to measure residual glycerol (Doyon, Gaudreau, Stgelais, Beaulieu, \& Randall, 1991). Results are presented as the average of triplicates $(n=3)$.

\subsection{Extraction of lipids}

Total lipid was extracted from a known weight (approximately 5.7 and $2.3 \mathrm{mg}$ for nitrate replete and deplete cultures, respectively) of freeze-dried biomass using the Folch method (Elahee Doomun et al., 2016). Lipid extracts were dried at $50{ }^{\circ} \mathrm{C}$ under $\mathrm{N}_{2}$ gas.

\subsection{Solid phase extraction}

Silica-based Solid phase extraction (SPE) cartridges $\left(\right.$ SampliQ $^{\mathrm{TM}}$, Agilent Technologies) were used to separate lipids into neutral lipid (TAG), glycolipid (GL) and phospholipid (PL) fractions. Lipid extracts were loaded on the silica columns after resuspending in $300 \mu \mathrm{L}$ of $1 \%$ acetic acid in chloroform. TAG, GL and PL fractions were sequentially eluted using $1 \%$ acetic acid in chloroform, acetone:methanol mixture (9:1 v/v) and methanol, respectively (Elahee Doomun et al., 2016). Internal standards for free fatty acid ( $\mathrm{d}_{27}$-myristic acid, Sigma Aldrich) and glycerol backbone ( $\mathrm{d}_{5}$-glycerol, Sigma Aldrich) were added to each fraction. Fractions were dried at $50^{\circ} \mathrm{C}$ under $\mathrm{N}_{2}$ gas.

This article is protected by copyright. All rights reserved. 


\subsection{Hydrolysis of lipid fractions}

Lipid fractions in Kimble tubes were hydrolysed into free fatty acids and lipidassociated glycerol backbone using $1 \mathrm{~mL}$ of $0.5 \mathrm{M} \mathrm{HCl}$ in acetonitrile:water solvent $(9: 1 \mathrm{v} / \mathrm{v})$. Samples were incubated at $100{ }^{\circ} \mathrm{C}$ for $45 \mathrm{~min}$ and cooled to room temperature. Free fatty acids and lipid-associated glycerol backbone were separated using $1 \mathrm{~mL}$ of hexane and $1 \mathrm{~mL}$ of de-ionised water. Samples were sonicated in an ultrasonic water bath for $30 \mathrm{~min}$ and centrifuged at $1000 \mathrm{~g}$ for $5 \mathrm{~min}$. The top hexane layer containing free fatty acids was transferred to new Kimble tubes. The fatty acids were re-extracted from the remaining hexane top layer by the further addition of hexane $(1 \mathrm{~mL})$. The bottom aqueous layer containing lipid-associated glycerol backbones was retained. Hexane and aqueous layers were dried at $50{ }^{\circ} \mathrm{C}$ and $70{ }^{\circ} \mathrm{C}$, respectively under $\mathrm{N}_{2}$ gas.

\subsection{Fatty acid analysis using $G C-M S$}

Pentaflurorobenzyl derivatives were prepared from the free fatty acids. The fatty acids were derivatised by adding $50 \mu \mathrm{L}$ of $1 \% \mathrm{~N}, \mathrm{~N}$-diisopropylethylamine in acetonitrile and $50 \mu \mathrm{L}$ of $1 \%$ 2,3,4,5,6-pentafluorobenzyl bromide in acetonitrile (Elahee Doomun et al., 2016). Derivatised samples were dried using a speed vacuum (Christ RVC 218) for $20 \mathrm{~min}$ at $30{ }^{\circ} \mathrm{C}$ and resuspended in $50 \mu \mathrm{L}$ of choloroform:methanol $(2: 1 \mathrm{v} / \mathrm{v})$. A Thermo Trace DSQ single quadrupole GC-MS (Thermo Scientific ${ }^{\mathrm{TM}}$, Waltham, MA, USA) with HP-88 GC column [(88\% - Cyanopropy) aryl-polysiloxane; Agilent Technologies] operated in negative ion chemical ionisation (NCI) mode (see Supplementary S.2 for instrument details). Peak areas were determined for each isotopologue of all fatty acids using Xcalibur $^{\mathrm{TM}}$ (Thermo Scientific, USA) Qual processing software. The peak areas were then used to calculate the amount of ${ }^{13} \mathrm{C}$ 
incorporation using the Nanchen et al. method for mass isotope distribution analysis (Nanchen, Fuhrer, \& Sauer, 2007). This enabled the quantification of the ${ }^{13} \mathrm{C}$ present in 22 individual fatty acids including correction for the presence of background ${ }^{13} \mathrm{C}$. Individual fatty acids were quantified using an external standard curve normalised to the $\mathrm{d}_{27}$-myristic acid internal standard.

\subsection{Lipid-associated glycerol backbone analysis using GC-MS}

Aqueous layers containing lipid-associated glycerol backbone were derivatised using $50 \mu \mathrm{L}$ of pyridine and $50 \mu \mathrm{L}$ of acetic anhydride and incubated at $60{ }^{\circ} \mathrm{C}$ for $30 \mathrm{~min}$. Derivatised samples were dried using a speed vacuum for $20 \mathrm{~min}$ at $30{ }^{\circ} \mathrm{C}$ and resuspended in $50 \mu \mathrm{L}$ of ethyl acetate. GC-MS operated in positive ion chemical ionization mode (PCI) with a VF- $5 \mathrm{~ms} 30 \mathrm{~m}, 0.25 \mathrm{~mm}, 0.25 \mu \mathrm{m}, \mathrm{GC}$ column with a 10 m EZ-Guard (Agilent Technologies) and methane $(2 \mathrm{~mL} / \mathrm{min})$ as a reagent gas (see Supplementary S.3 for instrument details). Selected ion monitoring (SIM) was performed a $159 \mathrm{~m} / \mathrm{z}\left(\mathrm{M}_{0}\right)$ to $164 \mathrm{~m} / \mathrm{z}$ to obtain the peak areas of both derivatised glycerol triacetate ion and $\mathrm{d}_{5}$-glycerol triacetate ion, respectively (Foletta et al., 2016). Glycerol in the samples was quantified using an unlabelled glycerol standard series normalised to the internal standard ( $\mathrm{d}_{5}$-glycerol).

\section{Results and Discussion}

Labelling experiments were conducted using ${ }^{13} \mathrm{C}$ stable isotopes to track the assimilation of glycerol carbon into fatty acyl chains and lipid-associated glycerol backbones of the different lipid fractions (TAG, GL, and PL). N. salina was grown mixotrophically using ${ }^{13} \mathrm{C}_{3^{-}}$glycerol under nitrate replete $(1.17 \mathrm{mM} ; \sim 14 \mathrm{mg}-\mathrm{N} / \mathrm{L})$ and deplete $(0 \mathrm{mM})$ conditions. Glycerol consumption was found to be $78.0 \pm 1.3 \%$ and $13.3 \pm 1.6 \%$ after 7 days under nitrate replete and nitrate deplete conditions, 
respectively. Under nitrate replete conditions, there was still nitrogen detected at the end of the cultivation period.

\subsection{Quantitative analysis of acyl content}

The fatty acyl chains $\left(\mu g_{\text {acyl chains }} / \mathrm{mg}_{\text {biomass }}\right)$ in the TAG, GL and PL fractions were quantified (Fig. 1) to determine the overall changes in the lipid profile resulting from nitrogen deprivation during mixotrophic growth on glycerol. The nitrate depleted culture had a higher cellular TAG content than the nitrate replete culture (51 \pm 3 and $38 \pm 1 \mu \mathrm{g}_{\text {acyl-chain }} / \mathrm{mg}_{\text {biomass, }}$ respectively) (Fig. 1). However, the reduced biomass productivity in the nitrate deplete culture $\left(8.5 \mathrm{mg}_{\text {drybiomass }} / \mathrm{L} / \mathrm{d}\right.$ compared to 30 $\mathrm{mg}_{\text {drybiomass }}(\mathrm{L} / \mathrm{d}$ in the nitrate replete culture) meant that the actual TAG and overall lipid productivities were lower $\left(0.9 \mathrm{mg}_{\text {lipid }} / \mathrm{L} / \mathrm{d}\right.$ compared to $13.1 \mathrm{mg}_{\text {lipid }} / \mathrm{L} / \mathrm{d}$ in the nitrate replete culture). This trade-off between TAG accumulation and biomass productivity during phototrophic growth is well known (Ajjawi et al., 2017). Unfortunately, it does not appear that supplementation of glycerol during nitrate depletion can resolve this issue for this strain of Nannochloropsis. Nonetheless, the TAG content of mixotrophic cultures of $N$. salina was increased by $\sim 33 \%$ under nitrate stress. Nitrate depletion decreased the cellular concentration of both GL fraction $(\sim 15 \%)$ and PL fraction $(\sim 44 \%)$.

Modifications in the acyl chain profiles were also analysed (Fig. 1). The prominent acyl chains in the TAG fraction under nitrate replete conditions were C14:0, C16:0, C16:1, C18:0, C18:1, and C20:5. Upon nitrogen deprivation, the increase in TAG was predominantly due to increases in $\mathrm{C} 16: 0$ and $\mathrm{C} 18: 0$ by $55 \%$ and $73 \%$, respectively. There was a slight decrease in the C14:0 (-16\%), and C18:3 (-47\%) acyl chains. In the GL fraction, the prominent acyl chains under nitrate replete conditions were C14:0, 
C16:0, C16:1, C18:0, C18:1, C18:2, C18:3 and C20:5. Upon nitrogen deprivation, there was decreases in the concentrations of $\mathrm{C} 14: 0(-21 \%), \mathrm{C} 16: 1(-11 \%), \mathrm{C} 18: 1(-$ 70\%), C18:3 (-65\%) and C20:5 (-3\%) approximately balanced by an increase in C18:0 (29\%) acyl chains. The prominent acyl chains in the PL fraction under nitrate replete conditions were $\mathrm{C} 14: 0, \mathrm{C} 16: 0, \mathrm{C} 16: 1, \mathrm{C} 18: 1, \mathrm{C} 18: 3$, and $\mathrm{C} 20: 5$. Upon nitrogen deprivation there was larger decreases in C14:0 (-52\%), C16:0 (-50\%), C16:1 (-37\%), C18:1 (-59\%), C18:3 (-47\%) and C20:5 (-22\%). The increase in C16:0 and C18:0 acyl chains in TAG under nitrate deplete conditions is consistent with a previously published investigation into de novo biosynthesis of these acyl chains during nitrogen deprivation (Martin et al., 2014). Nitrate deprivation had a minor effect on the C20:5 content of the GL and PL, but not in the TAG. A high content of polyunsaturated fatty acids (i.e. C20:5) usually reflects remodelling/recycling of phospholipids via acyl editing cycle reactions (Allen, DiRusso, \& Black, 2015). Moreover, it has previously been reported that biosynthesis of polar lipids was majorly unaffected by nitrate stress (Martin et al., 2014). This is consistent with the current results, in which the overall acyl chain profile of the GL and PL fractions remained relatively constant upon nitrogen deprivation (Figs. 1-b and -c).

\subsection{Fate of the ${ }^{13} \mathrm{C}$ in the consumed glycerol}

A complete carbon balance was not within the scope of the study. In addition to quantifying the ${ }^{13} \mathrm{C}$ in the consumed glycerol and algal lipids, this would require quantifying the ${ }^{12} \mathrm{C}$ carbon assimilated from $\mathrm{CO}_{2}$, the ${ }^{12} \mathrm{C}$ and ${ }^{13} \mathrm{C}$ content of the nonlipid biomass components, and the losses of ${ }^{12} \mathrm{C}$ and ${ }^{13} \mathrm{C}$ in the respired $\mathrm{CO}_{2}$ and extracellular products. Nonetheless, the proportion of the ${ }^{13} \mathrm{C}$ in the consumed glycerol that was incorporated into the acyl chain and glycerol backbones of the 
different lipid fractions could be determined (Table 1). Even after considering the large difference in the amount of glycerol consumed in the nitrate replete and deplete media ( $78 \%$ and $13 \%$, respectively), there was a dramatic difference in the utilisation of the consumed glycerol for lipid synthesis. Under both conditions, the majority of the ${ }^{13} \mathrm{C}$ in the lipids was found in the acyl chains of the membrane lipids (GL and PL) rather than in the TAG or glycerol backbones. The relative proportion of the consumed ${ }^{13} \mathrm{C}$ that ended up as lipid-associated glycerol backbone was reasonably similar between the nitrate replete and deplete cultures (Table 1). It was hypothesised that the addition of glycerol under nitrate deplete conditions would be utilised in TAG production by providing a glycerol backbone (in the form of glycerol-3-phosphate) on which acyl chains are added. However, only a small amount of glycerol (only $\sim 1.2 \%$ of the $13 \%$ of consumed glycerol), ended up as the backbone for the lipid fractions, with more being funnelled into de novo acyl chain synthesis, the majority of which ended up on the membrane lipids (Table 1). This contrasts with previous literature, in which the addition of glycerol was shown to induce TAG accumulation significantly in the diatom P. tricornutum (Villanova et al., 2017) and the oil-producing yeast Lipomyces starkeyi (Maruyama et al., 2018) by increasing intracellular carbon intermediates of the Calvin-Benson cycle.

As a source of reduced organic carbon and a building block of saponifiable lipids, glycerol can flow through several different metabolic pathways in various locations within an algal cell. As an uncharged molecule glycerol can first enter into the cytoplasm of the cell through passive diffusion (da Silva, Mack, \& Contiero, 2009). Here, the glycerol can enter central metabolism to be added to the carbon skeleton pool or be oxidised to $\mathrm{CO}_{2}$ for cellular energy during periods of dark respiration. Alternatively, the glycerol can be phosphorylated to glycerol-3-phosphate (G3P) 
(Perez-Garcia, Escalante, de-Bashan, \& Bashan, 2011) to become the backbone for lipid synthesis. This can include conversion to phospholipids and TAG via the endoplasmic reticulum (ER) or into glycolipids in the chloroplast (Martin et al., 2014). The appearance of ${ }^{13} \mathrm{C}$ in the glycerol backbones of the PL and GL fractions (Table 1), indicates that some glycerol went through both these pathways.

Further, an analysis of the ${ }^{13} \mathrm{C}$ labelling of the glycerol backbone (Fig. 2) shows a disproportionate amount of fully (triple ${ }^{13} \mathrm{C}$ ) labelling. The lipid-associated glycerol backbones being were predominantly ${ }^{13} \mathrm{C}_{3}$ labelled. This indicates that intact glycerol molecules were in fact directly esterified to fatty acids/polar head groups. The presence of singly and doubly labelled lipid-bound glycerol species suggests that a smaller percentage of the glycerol-3-phosphate destined for lipid esterification was formed from glycerol passed via the central carbon metabolism. The glycerol going into central carbon metabolism via G3P can be converted to glyceraldehyde-3phosphate (GAP) and dihydroxyacetone phosphate (DHAP) (intermediates of the Calvin-Benson cycle) and can enter the Embden-Meyerhof-Parnas (EMP) pathway as one of the intermediates of glycolysis. The lower glycolysis pathway will sequentially breakdown GAP to form acetyl-CoA (the precursor for fatty acid biosynthesis) and/or can enter in TCA (tricarboxylic acid) cycle to provide carbon skeletons for amino acids synthesis (Huang et al., 2015; Ryu et al., 2018). Additionally, the G3P can also be assimilated in the upper glycolysis pathway (gluconeogenesis) to store carbon reserves as carbohydrates (Villanova et al., 2017). However, in Nannochloropsis sp. only a small amount of carbohydrates (5-17\% dry weight basis) are synthesised compared to proteins (23-59\% dry weight basis) and lipids (9-62\% dry weight basis) (Subramanian, Barry, Pieris, \& Sayre, 2013). The appearance of a significant 
proportion of the consumed ${ }^{13} \mathrm{C}$ in the acyl chains of the lipids (Table 1) clearly shows that glycerol goes into de novo fatty acid synthesis within the chloroplast.

\section{$3.3{ }^{13}$ C labelling on acyl chains and glycerol backbone of different lipid fractions}

${ }^{13} \mathrm{C}$ labelling of the key acyl chains within the three lipid classes of $N$. salina (Fig. 3) was analysed (Elahee Doomun et al., 2016). In nitrate replete conditions, labelling of the acyl chains in the TAG fraction was in the range 2.2-82.1\%. Labelling of the GL fraction was in the range of $3.7-75.4 \%$. Labelling of the PL fraction was generally higher, in the range of 7.1-91.4\%. In nitrate deplete conditions, acyl chain labelling was generally much low due to the reduced consumption of ${ }^{13} \mathrm{C}_{3}$-glycerol. Labelling of TAG was in the range of 1.6-7.0\%. Labelling of the GL fraction was between 1.311.8\%. Labelling of the PL fraction was in the range 1.5-10.3\%. There was very little labelling in the C16:2 and C18:0 acyl chains of any lipid fraction under nitrate deplete conditions.

The proportion of the total carbon in the acyl chains that was ${ }^{13} \mathrm{C}$ was determined as a function of lipid fraction (Table 2) and type of acyl chain (Fig. 4). In terms of lipid fraction, there was a higher proportion of ${ }^{13} \mathrm{C}$ in the GL and PL than in the TAG under both replete and deplete conditions (Table 2). The distributions of acyl-incorporated ${ }^{13} \mathrm{C}$ amongst the major acyl chains in the various lipid fractions under nitrate replete and deplete conditions (Fig. 4) were reasonably consistent with the respective fatty acid profiles (Fig. 1). Under nitrate replete and deplete conditions, the relative proportion of ${ }^{13} \mathrm{C}$ was generally higher in de novo synthesised monounsaturated (e.g. C16:1 and C18:1) than the saturated C16:0 or polyunsaturated C20:5 (Fig. 4) acyl chains of TAG. This reflects the flux of carbon from glycerol going firstly to de novo 
fatty acid synthesis before acyl editing reactions (Allen et al., 2017). This is consistent with the highest extent of ${ }^{13} \mathrm{C}$ incorporation in the C18:1 acyl chains of the PL fraction (Fig. 4-c), which is one of the primary precursors for the acyl editing cycle reactions (Bates \& Browse, 2012).

\subsection{Carbon labelling patters within acyl chains}

This isotopomer labelling pattern within an acyl chain can reveal the sources of the carbon used to build it. For instance, a C16 fatty acid, when full labelled by ${ }^{13} \mathrm{C}$, has been entirely synthesised from carbon originating from the added glycerol to the media. The labelling patterns in the key saturated $(\mathrm{C} 16: 0)$ and polyunsaturated (C20:5) fatty acids within the different lipid fractions were analysed (Fig. 5). In the nitrate replete cultures, the labelling patterns for both C16:0 and C20:5 were generally parabolic in the mid to high range of ion abundance (roughly between $M+6$ and $\mathrm{M}+20$, respectively), which shows a mix of carbon sources used to build the acyl chains. There was slightly a different labelling pattern in PL C16:0, with a relatively high abundance of fully labelled carbon $(\mathrm{M}+16)$. In the nitrate deplete cultures, the detectable ion abundance displayed an exponential decay from $\mathrm{M}+0$ for both $\mathrm{C}$ 16:0 and C20:5 in all three lipid fractions clearly indicating limited uptake of glycerol. The relatively even spread of ${ }^{13} \mathrm{C}$ isotopologues show that the de novo acyl chains are a mixture of ${ }^{13} \mathrm{C}$ and ${ }^{12} \mathrm{C}$. This indicates that the acyl synthesis combines ${ }^{13} \mathrm{C}$ from glycerol that has be brought through to acetyl-CoA along with ${ }^{12} \mathrm{C}$ (which is presumably a mix of ${ }^{12} \mathrm{C}$ from $\mathrm{CO}_{2}$ fixed by photosynthesis during the mixotrophic cultivation period, and recycled carbon from the original inoculum). The high $\mathrm{M}+16$ peak (carbon entirely from glycerol) for the nitrate replete C16:0 could indicate a 
more direct funnelling of carbon from glycerol into C16:0 for some periods of the cultivation.

\subsection{Proposed carbon flux for fatty acid synthesis}

Although glycerol metabolism has not been exhaustively studied in $N$. salina, a general discussion is possible based on established knowledge. As mentioned above (see Section 3.2), glycerol is initially phosphorylated to G3P, which can enter the glycolysis pathway and/or be directly incorporated as the glycerol backbone of glycerolipids through sequential acylation. The major fatty acyl chains (C16:0, C18:0, C18:1, etc.) synthesised in the plastid are esterified by Coenzyme-A (CoA) to enter the acyl-CoA pool. The resulting acyl-CoA enters the ER for the acylation of G3P for the subsequent synthesis of lysophosphatidic acid (lyso-PA), phosphatidic acid (PA), de novo diacylglycerol (DAG) (Bates \& Browse, 2012) and TAG, completing the Kennedy pathway (Allen et al., 2017). However, there is also a possibility of TAG being synthesised with an unusual acyl chain profile including highly polyunsaturated acyl chains (as seen in the current study; Fig. 1-a). The enzymatic desaturation of saturated acyl chains of PL (for instance, PC-C18:1, produced from de novo DAG and/or the acyl-CoA pool via the acyl editing cycle) produces modified-PL (e.g. PCC18:2, -C18:3) with highly polyunsaturated acyl chains (Bates \& Browse, 2012), which serves as another substrate for TAG, resulting in a high degree of polyunsaturation. In addition to TAG, the results show a high content of polyunsaturated acyl chains in the GL fraction (Fig. 1-b). This leads to the assumption that hydrolysis of modified-PL (having highly polyunsaturated acyl chains) may serve as a substrate for synthesis of modified-GL in the chloroplast. If this is the case, then the overall ${ }^{13} \mathrm{C}$ incorporation and concentration of GL should be more than PL. 
Interestingly, this was observed in the current study where the total GL concentration was consistently higher than PL under both nitrate replete and deplete conditions (Table 2). It has been previously reported that the reverse reactions can then convert the modified-GL into TAG with a high level of polyunsaturation (Bates \& Browse, 2012). Further research is needed to analyse real-time carbon flux from GL/PL to TAG synthesis in Nannochloropsis.

Under both nitrate replete and deplete conditions, there was a less ${ }^{13} \mathrm{C}$ in the polyunsaturated acyl chains than in the monounsaturated acyl chains (Fig. 3). Additionally, the nitrate depleted cultures had higher labelling on PL-associated glycerol backbones compared to GL-associated glycerol backbones, while labelling on TAG-associated and GL-associated glycerol backbones were found to be similar (Fig. 2). This could suggest that nitrogen deprived conditions imbalanced/decreased the carbon flux from PL to GL for acyl editing reactions (polyunsaturation), while the carbon flux of PL was increased towards synthesis of TAG via the Kennedy pathway (Bates \& Browse, 2012). Nonetheless, based on the literature and the results of this study, a summary of the possible pathways for lipid synthesis from glycerol can be proposed (Fig. 6).

The current study shows the importance of nitrogen for efficient utilisation of glycerol and recommends against the use of mixotrophic cultivation under nitrate deprived conditions. It is possible that moderate nitrogen depletion under mixotrophic conditions could improve TAG production compared to fully nitrate depleted conditions (Paranjape et al., 2016a). Further isotopic labelling studies are recommended that consider the combination of labelled substrates (such as ${ }^{13} \mathrm{C}$ acetate and ${ }^{13} \mathrm{C}_{3}$-glycerol) with more frequent time-points to simultaneously study the 
initial carbon flux for protein and fatty acid synthesis (Juergens, Disbrow, \& ShacharHill, 2016). A more detailed picture of carbon flux during mixotrophic growth will help when genetically modifying the selected microalga to divert carbon towards TAG synthesis with less loss of carbon to other metabolites.

\section{Acknowledgements}

The authors are grateful to the University of Melbourne for a Melbourne International Engagement Award Scholarship for Ms. Poddar. The current research is the collaborative work between the University of Melbourne and Deakin University.

\section{Conflict of interest statement}

Authors declare no conflict of interest.

\section{References}

Ajjawi, I., Verruto, J., Aqui, M., Soriaga, L. B., Coppersmith, J., Kwok, K.,... Moellering, E. R. (2017). Lipid production in Nannochloropsis gaditana is doubled by decreasing expression of a single transcriptional regulator. Nature Biotechnology, 35(7), 647-652. doi:10.1038/nbt.3865

Allen, J. W., DiRusso, C. C., \& Black, P. N. (2015). Triacylglycerol synthesis during nitrogen stress involves the prokaryotic lipid synthesis pathway and acyl chain remodeling in the microalgae Coccomyxa subellipsoidea. Algal ResearchBiomass Biofuels and Bioproducts, 10, 110-120. doi:10.1016/j.algal.2015.04.019

Allen, J. W., DiRusso, C. C., \& Black, P. N. (2017). Carbon and acyl chain flux during stress-induced triglyceride accumulation by stable isotopic labeling of the polar microalga Coccomyxa subellipsoidea C169. Journal of Biological Chemistry, 292(1), 361-374. doi:10.1074/jbc.M116.760843

This article is protected by copyright. All rights reserved. 
Bates, P. D., \& Browse, J. (2012). The significance of different diacylgycerol synthesis pathways on plant oil composition and bioengineering. Frontiers in Plant Science, 3, 147. doi:10.3389/fpls.2012.00147

Bates, P. D., Durrett, T. P., Ohlrogge, J. B., \& Pollard, M. (2009). Analysis of acyl fluxes through multiple pathways of triacylglycerol synthesis in developing soybean embryos. Plant physiology, 150(1), 55-72. doi:10.1104/pp.109.137737

da Silva, G. P., Mack, M., \& Contiero, J. (2009). Glycerol: a promising and abundant carbon source for industrial microbiology. Biotechnology Advances, 27(1), 3039. doi:10.1016/j.biotechadv.2008.07.006

Deschenes, J. S., Boudreau, A., \& Tremblay, R. (2015). Mixotrophic production of microalgae in pilot-scale photobioreactors: Practicability and process considerations. Algal Research-Biomass Biofuels and Bioproducts, 10, 80-86. doi:10.1016/j.algal.2015.04.015

Doyon, G., Gaudreau, G., Stgelais, D., Beaulieu, Y., \& Randall, C. J. (1991). Simultaneous HPLC determination of organic-acids, sugars and alcohols. Canadian Institute of Food Science and Technology Journal-Journal De L Institut Canadien De Science Et Technologie Alimentaires, 24(1-2), 87-94. doi:Doi 10.1016/S0315-5463(91)70025-4

Ecker, J., \& Liebisch, G. (2014). Application of stable isotopes to investigate the metabolism of fatty acids, glycerophospholipid and sphingolipid species. Progress in Lipid Research, 54, 14-31. doi:10.1016/j.plipres.2014.01.002

Elahee Doomun, S. N., Loke, S., O'Callaghan, S., \& Callahan, D. L. (2016). A simple method for measuring carbon-13 fatty acid enrichment in the major lipid classes of microalgae using GC-MS. Metabolites, 6(4), 1-13. doi:10.3390/metabo6040042

Foletta, V. C., Palmieri, M., Kloehn, J., Mason, S., Previs, S. F., McConville, M. J.,... Kowalski, G. M. (2016). Analysis of mammalian cell proliferation and 
macromolecule synthesis using deuterated water and gas chromatographymass spectrometry. Metabolites, 6(4). doi:10.3390/metabo6040034

Huang, A., Liu, L., Yang, C., \& Wang, G. (2015). Phaeodactylum tricornutum photorespiration takes part in glycerol metabolism and is important for nitrogen-limited response. Biotechnology for Biofuels, 8(1), 73. doi:10.1186/s13068-015-0256-5

Janssen, J. H., Lamers, P. P., de Vos, R. C. H., Wijffels, R. H., \& Barbosa, M. J. (2019). Translocation and de novo synthesis of eicosapentaenoic acid (EPA) during nitrogen starvation in Nannochloropsis gaditana. Algal ResearchBiomass Biofuels and Bioproducts, 37, 138-144. doi:10.1016/j.algal.2018.11.025

Juergens, M. T., Disbrow, B., \& Shachar-Hill, Y. (2016). The relationship of triacylglycerol and starch accumulation to carbon and energy flows during nutrient deprivation in Chlamydomonas reinhardtii. Plant physiology, 171(4), 2445-2457. doi:10.1104/pp.16.00761

Martin, G. J., Hill, D. R., Olmstead, I. L., Bergamin, A., Shears, M. J., Dias, D. A.,... Callahan, D. L. (2014). Lipid profile remodeling in response to nitrogen deprivation in the microalgae Chlorella sp. (Trebouxiophyceae) and Nannochloropsis sp. (Eustigmatophyceae). PLoS One, 9(8), e103389. doi:10.1371/journal.pone.0103389

Maruyama, Y., Toya, Y., Kurokawa, H., Fukano, Y., Sato, A., Umemura, H.,... Shimizu, H. (2018). Characterization of oil-producing yeast Lipomyces starkeyi on glycerol carbon source based on metabolomics and ${ }^{13} \mathrm{C}$-labeling. Applied Microbiology and Biotechnology, 102(20), 8909-8920. doi:10.1007/s00253-018-9261-5

Nanchen, A., Fuhrer, T., \& Sauer, U. (2007). Determination of metabolic flux ratios from ${ }^{13} \mathrm{C}$-experiments and gas chromatography-mass spectrometry data Metabolomics: Methods and Protocols (pp. 177-197). Totowa, NJ: Humana Press.

This article is protected by copyright. All rights reserved. 
Pagnanelli, F., Altimari, P., Trabucco, F., \& Toro, L. (2014). Mixotrophic growth of Chlorella vulgaris and Nannochloropsis oculata: interaction between glucose and nitrate. J Chem Technol Biotechnol, 89(5), 652-661. doi:10.1002/jctb.4179

Paranjape, K., Leite, G. B., \& Hallenbeck, P. C. (2016a). Effect of nitrogen regime on microalgal lipid production during mixotrophic growth with glycerol. Bioresource Technology, 214, 778-786. doi:10.1016/j.biortech.2016.05.020

Paranjape, K., Leite, G. B., \& Hallenbeck, P. C. (2016b). Strain variation in microalgal lipid production during mixotrophic growth with glycerol. Bioresour Technol, 204, 80-88. doi:10.1016/j.biortech.2015.12.071

Perez-Garcia, O., Escalante, F. M., de-Bashan, L. E., \& Bashan, Y. (2011). Heterotrophic cultures of microalgae: metabolism and potential products. Water Research, 45(1), 11-36. doi:10.1016/j.watres.2010.08.037

Poddar, N., Sen, R., \& Martin, G. J. O. (2018). Glycerol and nitrate utilisation by marine microalgae Nannochloropsis salina and Chlorella sp and associated bacteria during mixotrophic and heterotrophic growth. Algal ResearchBiomass Biofuels and Bioproducts, 33, 298-309. doi:10.1016/j.algal.2018.06.002

Poddar, N., Sen, R., \& Martin, G. J. O. (2019). Bacterial abundance and diversity in Microchloropsis salina (formerly Nannochloropsis salina) cultures in response to the presence of ammonium, nitrate and glycerol. Journal of Applied Phycology. doi:10.1007/s10811-019-02007-0

Pollard, M., Delamarter, D., Martin, T. M., \& Shachar-Hill, Y. (2015). Lipid labeling from acetate or glycerol in cultured embryos of Camelina sativa seeds: A tale of two substrates. Phytochemistry, 118, 192-203. doi:10.1016/j.phytochem.2015.07.021

Ryu, K. H., Sung, M. G., Kim, B., Heo, S., Chang, Y. K., \& Lee, J. H. (2018). A mathematical model of intracellular behavior of microalgae for predicting growth and intracellular components syntheses under nutrient-replete and -

This article is protected by copyright. All rights reserved. 
deplete conditions. Biotechnology and Bioengineering, 115(10), 2441-2455. doi:10.1002/bit.26744

Simionato, D., Basso, S., Giacometti, G. M., \& Morosinotto, T. (2013). Optimization of light use efficiency for biofuel production in algae. Biophysical Chemistry, 182, 71-78. doi:10.1016/j.bpc.2013.06.017

Subramanian, S., Barry, A. N., Pieris, S., \& Sayre, R. T. (2013). Comparative energetics and kinetics of autotrophic lipid and starch metabolism in chlorophytic microalgae: implications for biomass and biofuel production. Biotechnology for Biofuels, 6(1), 150-161. doi:10.1186/1754-6834-6-150

Villanova, V., Fortunato, A. E., Singh, D., Bo, D. D., Conte, M., Obata, T.,... Finazzi, G. (2017). Investigating mixotrophic metabolism in the model diatom Phaeodactylum tricornutum. Philosophical Transaction Royal Society B, 372(1728). doi:10.1098/rstb.2016.0404

Wang, D., Ning, K., Li, J., Hu, J., Han, D., Wang, H.,.. Xu, J. (2014). Nannochloropsis genomes reveal evolution of microalgal oleaginous traits. PLoS Genetics, 10(1), e1004094. doi:10.1371/journal.pgen.1004094

Xue, L. L., Chen, H. H., \& Jiang, J. G. (2017). Implications of glycerol metabolism for lipid production. Progress in Lipid Research, 68, 12-25. doi:10.1016/j.plipres.2017.07.002

Zhang, X., Misra, A., Nargund, S., Coleman, G. D., \& Sriram, G. (2018). Concurrent isotope-assisted metabolic flux analysis and transcriptome profiling reveal responses of poplar cells to altered nitrogen and carbon supply. The Plant Journal, 93(3), 472-488. doi:10.1111/tpj.13792

This article is protected by copyright. All rights reserved. 
Table 1 Percentage of the ${ }^{13} \mathrm{C}$ in the glycerol consumed by Nannochloropsis salina that was assimilated into various lipid components during nitrate replete and deplete mixotrophic growth. Results are presented as the average and standard deviation of triplicates or duplicates $(\dagger)$

\begin{tabular}{ccc}
\hline & $\begin{array}{c}\text { Percent of consumed }{ }^{13} \mathrm{C} \\
\text { assimilated as lipid acyl chains }\end{array}$ & $\begin{array}{c}\text { Percent of consumed }{ }^{13} \mathrm{C} \\
\text { assimilated as lipid glycerol }\end{array}$ \\
Lipid fraction & $(\%)$ & backbone (\%)
\end{tabular}

Nitrate replete Nitrate deplete Nitrate replete Nitrate deplete

\begin{tabular}{ccccc}
\hline TAG & $2.6 \pm 0.3$ & $0.97 \pm 0.02$ & $0.048 \pm 0.001$ & $0.095 \pm 0.002$ \\
Glycolipid & $19.6 \pm 0.6$ & $6.9 \pm 0.3$ & $0.71 \pm 0.03$ & $0.80 \pm 0.02^{\dagger}$ \\
Phospholipid & $19.5 \pm 1.1$ & $3.6 \pm 1.8$ & $0.55 \pm 0.03$ & $0.25 \pm 0.01^{\dagger}$ \\
\hline Sum & $41.8 \pm 1.3$ & $11.4 \pm 1.5$ & $1.31 \pm 0.02$ & $1.14 \pm 0.01$ \\
\hline
\end{tabular}

Table 2 Amount of incorporated ${ }^{13} \mathrm{C}$ within different lipid fractions. Results are presented as the average and standard deviation of triplicates

\section{TAG Glycolipid Phospholipid}

\begin{tabular}{ccccc} 
& $\begin{array}{c}\text { Proportion of acyl carbon as }{ }^{13} \mathrm{C} \\
\text { Nitrate }\end{array}$ & $41.7 \pm 2.9$ & $43.7 \pm 0.6$ & $80 \pm 1$ \\
replete & $(\%)$ & & \\
\cline { 2 - 4 } & & & & \\
& Total ${ }^{13} \mathrm{C}_{\text {incorporated }}$ & $12.1 \pm 1.3$ & $90 \pm 3$ & $90 \pm 5$ \\
\hline
\end{tabular}

This article is protected by copyright. All rights reserved. 
$\left(\mu \mathrm{g}_{13 \mathrm{C}} / \mathrm{mg}_{\text {biomass }}\right)$

Proportion of acyl carbon as ${ }^{13} \mathrm{C}$

$3.6 \pm 0.2 \quad 5.6 \pm 0.3 \quad 8.1 \pm 0.2$

$(\%)$

Nitrate

deplete

$$
\begin{gathered}
\text { Total }{ }^{13} \mathrm{C}_{\text {incorporated }} \\
\left(\mu \mathrm{g}_{13 \mathrm{C}} / \mathrm{mg}_{\text {biomass }}\right)
\end{gathered}
$$

$1.38 \pm$

$9.9 \pm 0.4 \quad 5.1 \pm 2.6$

\section{Figures}

Fig. 1 Concentrations of individual acyl chains within different lipid fractions of Nannochloropsis salina grown mixotrophically with glycerol under nitrate replete and deplete conditions. The results and error bars are presented as the average and standard deviation of measurements of triplicates.

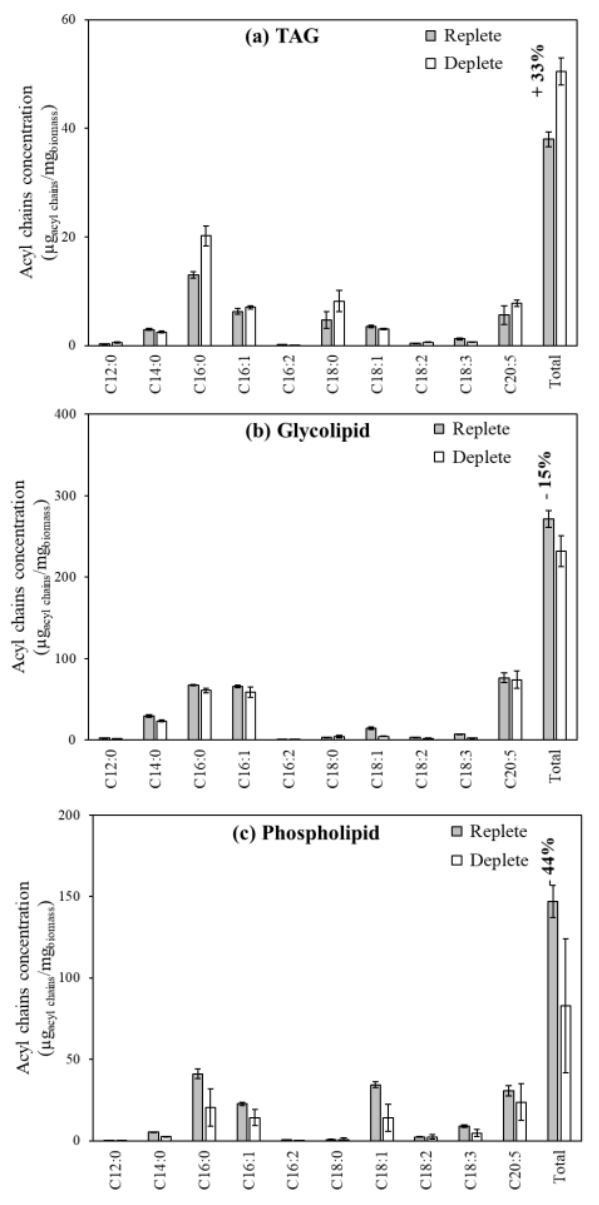

This article is protected by copyright. All rights reserved. 
Fig. $2{ }^{13} \mathrm{C}$ labelling (\% of carbon that is ${ }^{13} \mathrm{C}$ ) on lipid-associated glycerol backbones recovered from Nannochloropsis salina grown in $0.5 \mathrm{~g} / \mathrm{L}{ }^{13} \mathrm{C}_{3}$-glycerol under nitrate replete and deplete conditions. The results are presented as the average of triplicates or duplicates $(*)$.

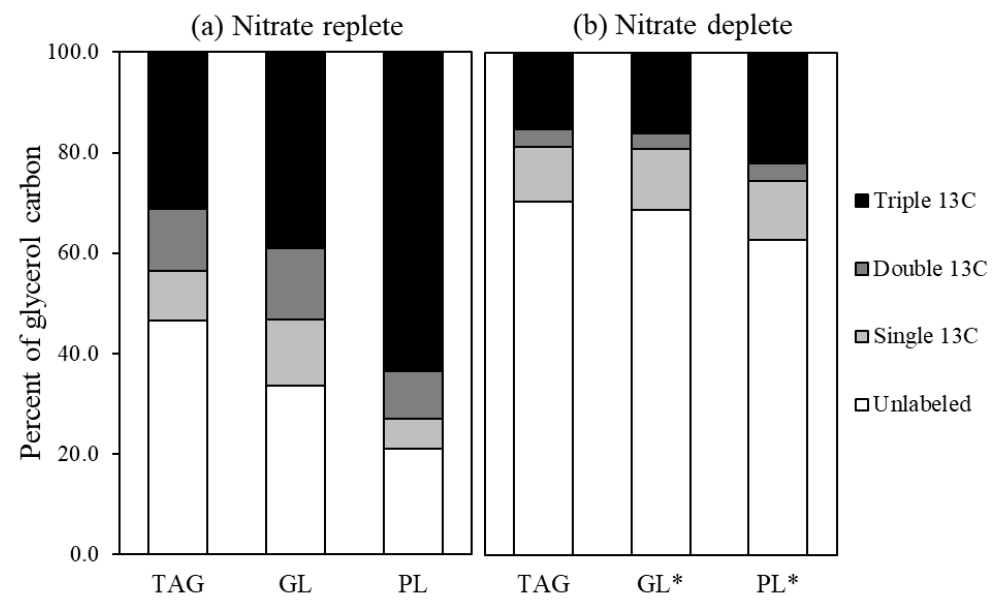

Fig. 3 Amount of ${ }^{13} \mathrm{C}$ labelling (\% of carbon that is ${ }^{13} \mathrm{C}$ ) on different acyl chains within TAG (filled bars), glycolipid (empty bars) and phospholipid (diagonally filled bars) fractions of Nannochloropsis salina grown with $0.5 \mathrm{~g} / \mathrm{L}{ }^{13} \mathrm{C}_{3}$-glycerol under (a) nitrate replete and (b) nitrate deplete conditions for 7 days. The results and error bars are presented as the average and standard deviation of triplicates.
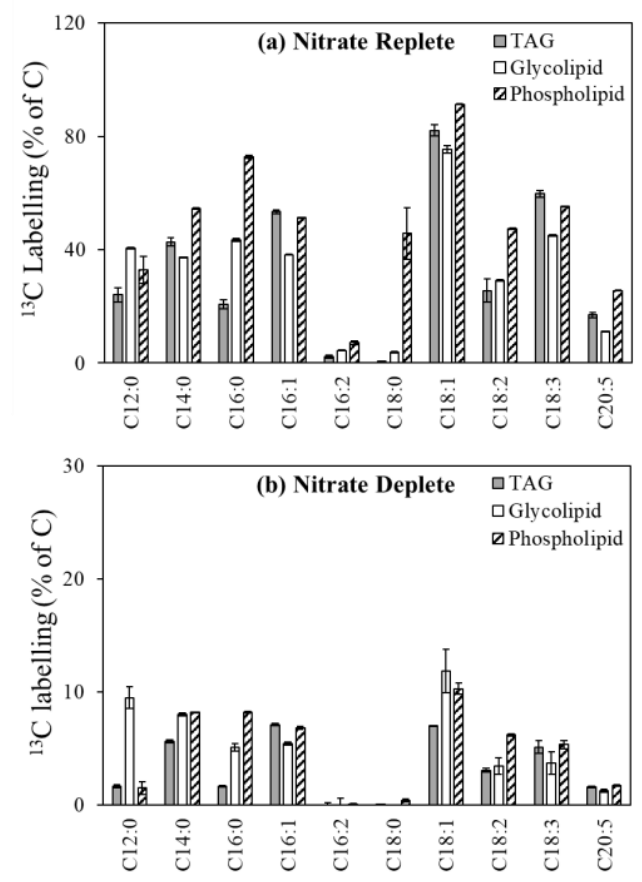

This article is protected by copyright. All rights reserved. 
Fig. 4 Proportion of the total carbon that is ${ }^{13} \mathrm{C}$ amongst the major acyl chains (\% of total) in the TAG, glycolipid, and phospholipid fractions of Nannochloropsis salina grown under nitrate replete and nitrate deplete conditions. The results and error bars are presented as the average and standard deviation of triplicates.
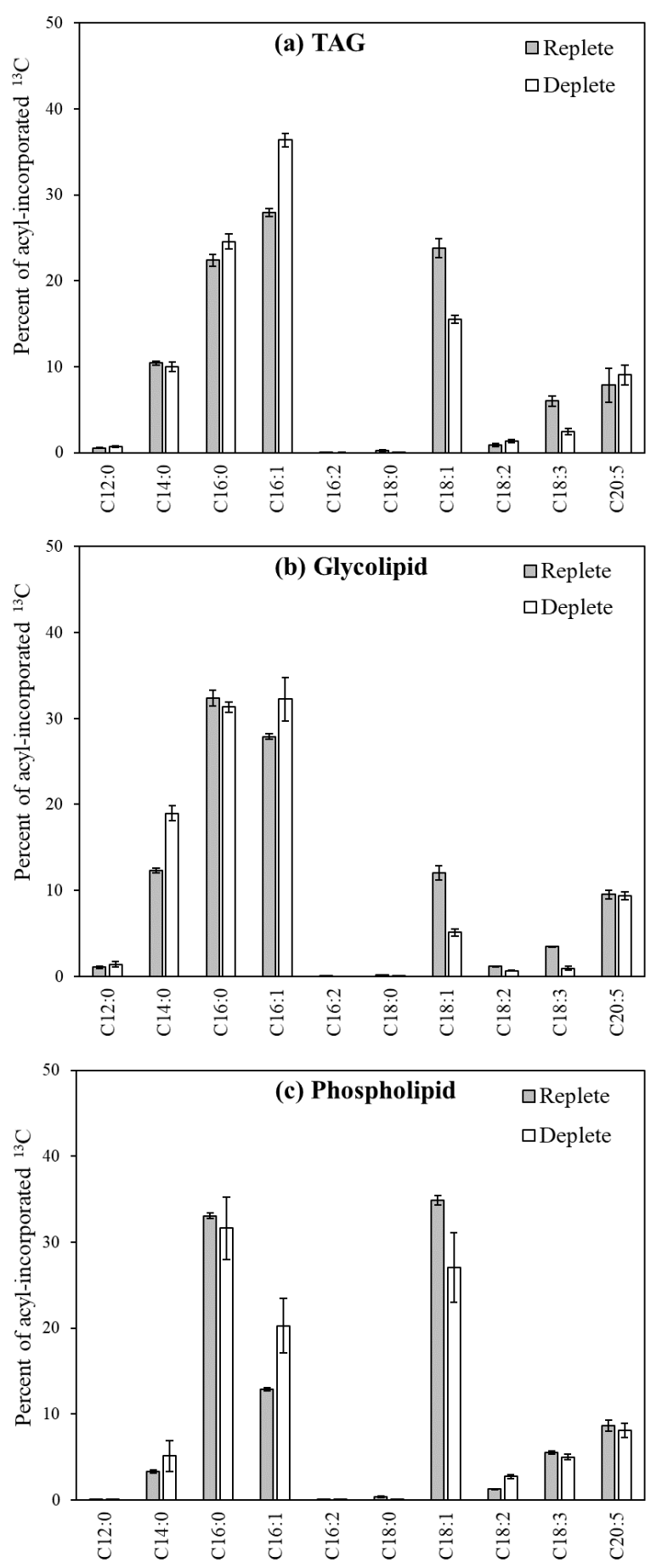

This article is protected by copyright. All rights reserved. 
Fig. $5{ }^{13} \mathrm{C}$ isotopologue distribution of unsaturated fatty acids within lipid classes of Nannochloropsis salina grown mixotrophically with ${ }^{13} \mathrm{C}_{3}$-glycerol under nitrate replete and deplete conditions for 7 days. The molecular ion signal (peak area) of each isotopologue $(M+n$; where $M$ is monoisotopic mass and $n$ is number of incorporated ${ }^{13} \mathrm{C}$ ) was normalised to the ion signal (peak area) of the internal standard and the algal biomass. The results and error bars are presented as the average and standard deviation of triplicate cultures.
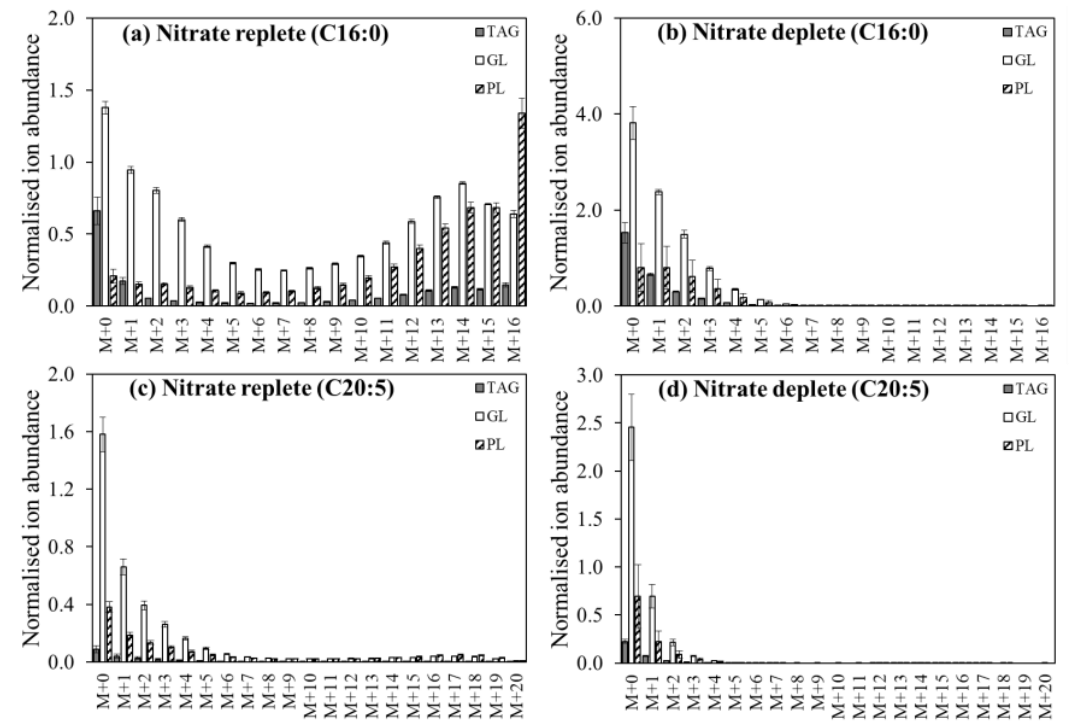

Fig. 6 (a) Possible fates of glycerol in algal metabolism. (b) Possible pathways for TAG synthesis through the Kennedy pathway and acyl editing reactions. Abbreviations: G3P, glycerol-3-phosphate; PA, phosphatidic acid; DAG, diacylglycerol; PC, phosphocholine; TAG, triacylglyceride; GL, glycolipids; PL, phospholipids; DHAP, dihydroxyacetone phosphate; GAP, glyceraldehyde phosphate; 3PGA, 3-phosphoglycerate; ER, endoplasmic reticulum; FA, fatty acid. Bold red text represents metabolites determined in Nannochloropsis salina mixotrophic cultures supplied with $0.5 \mathrm{~g} / \mathrm{L}{ }^{13} \mathrm{C}_{3}$-glycerol (this study). The amount of ${ }^{13} \mathrm{C}\left(\mu \mathrm{g}_{13 \mathrm{C}} / \mathrm{mg}_{\text {biomass }}\right.$ ) 
incorporated into the acyl chains of different lipid fractions under nitrate replete (NR) and nitrate deplete (ND) conditions are presented.

a)
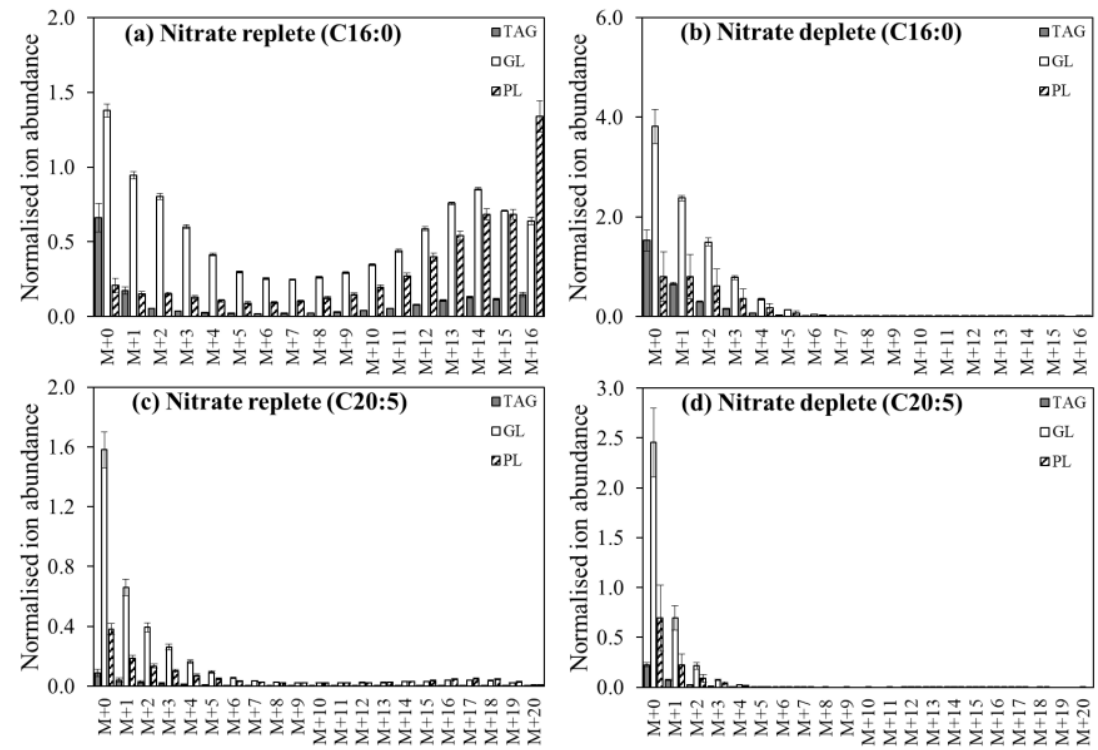

b)

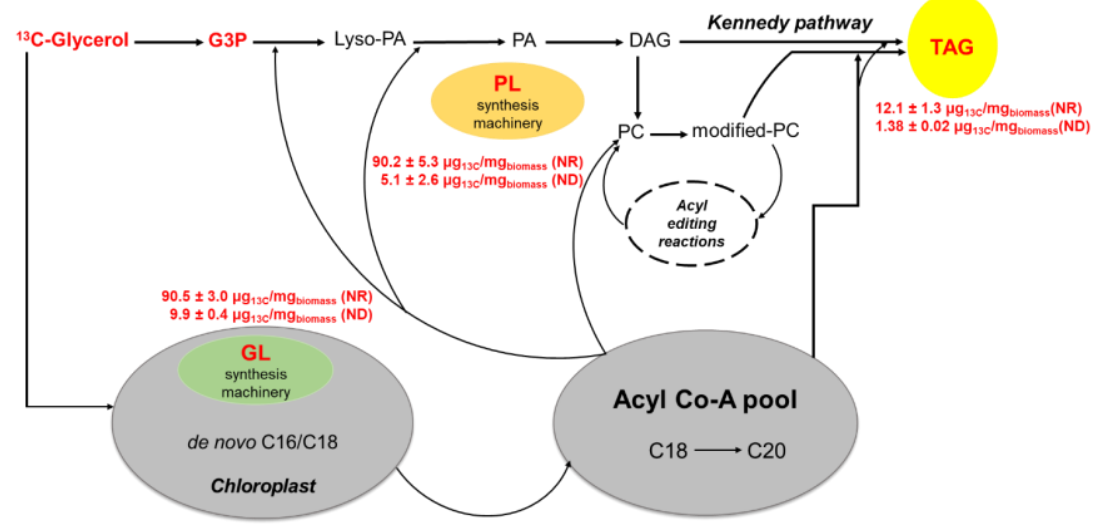

This article is protected by copyright. All rights reserved. 


\section{University Library}

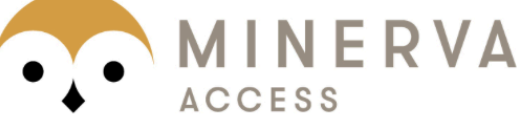

A gateway to Melbourne's research publications

Minerva Access is the Institutional Repository of The University of Melbourne

Author/s:

Poddar, N;Elahee Doomun, SN;Callahan, DL;Kowalski, GM;Martin, GJO

Title:

The assimilation of glycerol into lipid acyl chains and associated carbon backbones ofNannochloropsis salinavaries under nitrogen replete and deplete conditions

Date:

2020-11

Citation:

Poddar, N., Elahee Doomun, S. N., Callahan, D. L., Kowalski, G. M. \& Martin, G. J. O. (2020). The assimilation of glycerol into lipid acyl chains and associated carbon backbones ofNannochloropsis salinavaries under nitrogen replete and deplete conditions. Biotechnology and Bioengineering, 117 (11), pp.3299-3309. https://doi.org/10.1002/ bit.27498.

Persistent Link:

http://hdl.handle.net/11343/276013 